\title{
Clinical Study \\ Clinical Effectiveness of Peramivir in Comparison with Other Neuraminidase Inhibitors in Pediatric Influenza Patients
}

\author{
Toshiyuki Hikita, Hiroyuki Hikita, Fusako Hikita, Naoko Hikita, and Shizue Hikita
}

Pediatric Division, Hikita Pediatric Clinic, 2-7-20 Nakamachi, Kiryu City, Gunma 376-0035, Japan

Correspondence should be addressed to Toshiyuki Hikita, t-hikita@ra2.so-net.ne.jp

Received 30 October 2011; Accepted 16 December 2011

Academic Editor: Lavjay Butani

Copyright (๑) 2012 Toshiyuki Hikita et al. This is an open access article distributed under the Creative Commons Attribution License, which permits unrestricted use, distribution, and reproduction in any medium, provided the original work is properly cited.

\begin{abstract}
The currently used antivirals in the treatment of influenza in Japan include amantadine, oseltamivir, zanamivir, laninamivir, and peramivir. We compared the efficacy of intravenous peramivir with that of other neuraminidase inhibitors for treating pediatric influenza. The present study included 223 influenza patients ( $\leq 18$ years) who presented at the Hikita Pediatric Clinic between February and April 2011. We compared fever duration after starting treatment with antiviral drugs. Because inhalation drugs are difficult to use in $<5$-year-old patients and because of the potential adverse effects of oseltamivir in teenagers, we created two different age groups ( $<10$-year-old group and 5-18-year-old group) to evaluate treatment results. In influenza A patients between 5 and 18 years old, the median fever duration after treatment with zanamivir was 2 days, compared with 1 day for peramivir $(P=0.0242)$. In influenza B patients between 5 and 18 years old, the median fever duration after treatment with laninamivir was 3 days, compared with 1 day for peramivir $(P=0.0097)$. We found no significant difference for any of the other combinations of drug/disease type/age groups. No adverse effects were observed with the antiviral drugs used. The results suggest that peramivir is very useful in pediatric influenza patients.
\end{abstract}

\section{Introduction}

Of the various respiratory diseases, influenza is a major cause of mortality and morbidity among patients, particularly the very young and the elderly $[1,2]$. Two options are available for moderating the effect of the influenza virus: vaccines, which although effective, are underutilized and not completely protective because of frequent antigenic shifts in the viral surface proteins and antiviral drugs [2, 3]. Antiviral drugs have emerged as attractive options in the battle against influenza. Amantadine, oseltamivir, zanamivir, laninamivir, and peramivir are the five antiviral drugs currently used to treat influenza in Japan [2]. However, firm guidelines for prescribing these drugs remain to be established.

Amantadine is limited in effectiveness because of its lack of activity against influenza B virus [4] and the rapid emergence of resistant viral strains. Hemagglutinin and neuraminidase, two glycoproteins present on the viral surface, have antiviral targets [5]. Recently, oseltamivir and zanamivir, two influenza neuraminidase inhibitors, have commonly been prescribed for influenza A and B [6-12]. Laninamivir is a long-acting neuraminidase inhibitor for the treatment of influenza. A single inhalation of laninamivir is effective for the treatment of influenza, including that caused by the oseltamivir-resistant viruses, in adults $[13,14]$. However, seriously ill and pediatric patients need a parenteral formulation because the injectable drug is much easier to administer in such cases than oral oseltamivir, inhaled zanamivir, or laninamivir.

In Japan, peramivir has recently been approved for use not only in adults but also in children over 1 month of age [15]. In this study, we compared the efficacy of intravenous peramivir with that of other neuraminidase inhibitors for treating influenza infections in pediatric patients.

\section{Material and Methods}

The present study included 223 patients under the age of 18 years diagnosed with influenza at the Hikita Pediatric Clinic between February and April 2011. The patients presented 
TABLE 1: Comparison of the effectiveness of oseltamivir, zanamivir, laninamivir, and peramivir against influenza virus infection.

\begin{tabular}{|c|c|c|c|c|c|c|c|}
\hline \multicolumn{2}{|c|}{ Groups } & \multirow{2}{*}{ Therapy } & \multirow{2}{*}{$\begin{array}{l}\text { Number } \\
\text { of patients }\end{array}$} & \multirow{2}{*}{$\begin{array}{l}\text { Median age in } \\
\text { months (range) }\end{array}$} & \multirow{2}{*}{$\begin{array}{l}\text { Duration of fever } \\
\text { before treatment, } \\
\text { median day (range) }\end{array}$} & \multirow{2}{*}{$\begin{array}{l}\text { Duration of fever } \\
\text { after treatment, } \\
\text { median day (range) }\end{array}$} & \multirow{2}{*}{$P$ value } \\
\hline Influenza type & Ages (years) & & & & & & \\
\hline \multirow{2}{*}{ Influenza A } & \multirow[b]{2}{*}{$0-9$} & Oseltamivir & 83 & $51(3-118)$ & $1(0-2)$ & $2(0-6)$ & 0.4499 \\
\hline & & Peramivir & 22 & $41.5(2-106)$ & $1(0-2)$ & $1.5(1-3)$ & \\
\hline \multirow{2}{*}{ Influenza B } & \multirow{2}{*}{$0-9$} & Oseltamivir & 41 & $81(25-118)$ & $1(0-2)$ & $2(0-4)$ & 0.6435 \\
\hline & & Peramivir & 13 & $75(10-118)$ & $0(0-2)$ & $2(1-4)$ & \\
\hline \multirow{3}{*}{ Influenza A } & \multirow{3}{*}{$5-18$} & Laninamivir & 1 & $80(80-80)$ & $0(0-0)$ & $1(1-1)$ & $\begin{array}{c}\text { Not } \\
\text { performed }\end{array}$ \\
\hline & & Peramivir & 15 & $94(72-219)$ & $1(0-2)$ & $1(1-2)$ & \\
\hline & & Zanamivir & 18 & $34.5(69-198)$ & $1(0-2)$ & $2(0-3)$ & $0.0242^{*}$ \\
\hline \multirow{3}{*}{ Influenza B } & \multirow{3}{*}{$5-18$} & Laninamivir & 13 & $134(91-164)$ & $0(0-2)$ & $3(1-5)$ & 0.0097* \\
\hline & & Peramivir & 13 & $98(63-167)$ & $1(0-1)$ & $1(1-4)$ & \\
\hline & & Zanamivir & 20 & $120(87-179)$ & $0(0-2)$ & $2(0-4)$ & 0.2979 \\
\hline
\end{tabular}

"Not performed" indicates that statistical analysis was not performed because the number of subjects was too small.

* indicates a significant difference between peramivir and the other administered drug.

(Both the 0-9 years group and the 5-18 years group included 5-9-year-old children treated with peramivir).

with a complaint of fever lasting for less than $48 \mathrm{~h}$, and they were clinically diagnosed with rapid diagnostic tests. Specimens from nasal swabs or nasal aspirates were subjected to antigen detection. Commercial antigen detection kits based on immunochromatography (The Quick Chaser Flu A, B rapid antigen test [Mizuho Medy Co., Ltd. Saga, Japan]) was used for the diagnosis of influenza A or B. Subsequently, after obtaining informed consent from the parents, 35 patients diagnosed with influenza $\mathrm{A}$ by the rapid antigen $\mathrm{A}$, B test underwent a 2009 influenza A H1N1 virus infection test using the Quick Chaser Flu AH1pdm (Mizuho Medy) to differentiate patients with $2009 \mathrm{H} 1 \mathrm{~N} 1$ influenza from those with seasonal influenza.

The efficacy, potential adverse effects, and convenience of administration of the five antiviral drugs were explained to the patients and/or their families prior to study initiation. The choice of antivirals for influenza treatment was then discussed, and after obtaining informed consent from patients and/or their families, all patients underwent antiviral therapy. Laninamivir was administered as a single-inhalation dose of $40 \mathrm{mg}$ for patients aged $\geq 10$ years or $20 \mathrm{mg}$ for patients aged $<10$ years. Peramivir was administered intravenously as a single dose of $10 \mathrm{mg} / \mathrm{kg} / \mathrm{dose}$ (maximum $300 \mathrm{mg}$ / dose) over a period of $15 \mathrm{~min}$. Oral oseltamivir was prescribed twice per day in divided dosages for 5 days $(4 \mathrm{mg} /$ $\mathrm{kg} /$ day, maximum of $150 \mathrm{mg} /$ day). Zanamivir was administered twice per day at an inhalation dosage of $20 \mathrm{mg} /$ day for 5 days.

In 2007, the Ministry of Health, Labor, and Welfare, Japan, issued emergency instructions suspending the use of oseltamivir in patients aged 10-19 years [16]. Accordingly, oseltamivir was not prescribed for the teenage patients with influenza in our study. In addition, inhaled drugs are difficult to use in infants. Therefore, laninamivir and zanamivir were prescribed for patients aged $\geq 5$ years. We created 2 age groups for statistical analysis: patients aged $<10$ years and those aged between 5 and 18 years. We compared the duration of fever after initiating antiviral therapy. Fever was considered positive if the patient's body temperature was $\geq 37.5^{\circ} \mathrm{C}$. When a patient's body temperature dropped and remained at $<37.5^{\circ} \mathrm{C}$ for 48 continuous $\mathrm{h}$, the fever was considered as resolved.

Data were analyzed using JMP software version 8.0.2 (SAS Institute, Cary, NC, USA). The Wilcoxon rank-sum test and/or the Kruskal-Wallis rank-sum test were used to compare the ages of influenza patients treated with laninamivir, oseltamivir, peramivir, or zanamivir. We used KaplanMeier analysis and the log-rank test to compare the duration of fever after antiviral therapy initiation. A $P$ value of $<0.05$ was considered statistically significant.

\section{Results}

We treated 131 (70 males) and 92 (53 males) patients with influenza A and B, respectively. Nine of $35(25.7 \%)$ patients tested positive in the 2009 influenza A H1N1 test. Patients were administered the following antiviral drugs: laninamivir $(n=14)$, oseltamivir $(n=125)$, peramivir $(n=45)$, and zanamivir $(n=39)$. No patient was treated with amantadine in this study. The median ages of the patients treated with laninamivir, oseltamivir, peramivir, and zanamivir were 132.5 months (range, 80-164), 56.5 months (range, 3-135), 76 months (range, 2-219), and 124.5 months (range, 59198), respectively. No adverse effects were observed with any of the antiviral drugs used in this study.

We compared $<10$-year-old influenza patients who were administered either oseltamivir or peramivir (Table 1). The age of patients treated with oseltamivir was not significantly different from that of patients treated with peramivir. We also compared 5-18-year-old influenza patients treated with peramivir, zanamivir, or laninamivir. The age of patients did not significantly differ between treatment groups. The median 
duration of fever after zanamivir treatment in 5-18-year-old patients with influenza A was 2 days (range, 0-3 days), whereas that after peramivir treatment was 1 day (range, 12 days); this difference was statistically significant $(P=$ 0.0283 ). The median duration of fever after laninamivir treatment in 5-18-year-old patients with influenza B was 3 days (range, 1-5 days), whereas that after peramivir treatment was 1 day (range, $1-4$ days); this difference was also statistically significant $(P=0.0097)$. No other significant differences were observed for any of the other drug/disease type/age group combinations.

\section{Discussion}

Hernandez et al. reported their clinical experience with children $(n=11)$ hospitalized for 2009 influenza A (H1N1) and treated with peramivir [17]. In their study, all patients had rapidly progressing, radiographically confirmed viral pneumonia with respiratory failure. In our study, peramivir was administered to patients with influenza, including 2009 influenza A (H1N1), seasonal influenza H3N2, and influenza B. None of the patients had severe disease and all were treated as outpatients. Severe adult influenza infection has been reportedly treated with peramivir [18]. Although oseltamivir and zanamivir are used for severe influenza infections such as encephalopathy [19], peramivir is more suitable for such infections because it is much easier to administer (intravenously) to a severe case when compared with oral oseltamivir or inhaled zanamivir. Currently, most patients in Japan with an influenza-like illness are tested using rapid diagnostic tests and treated with an appropriate choice of antiviral drugs if results prove positive [20]. Particularly, after the $2009 \mathrm{H} 1 \mathrm{~N} 1$ influenza pandemic virus was isolated, antiviral therapy was recommended for all pediatric influenza patients.

A limitation of this study was the small study sample and the lack of a randomized open label study; therefore, more data are needed before the clinical implications of this study become clear. We compared only fever duration and did not analyze any other symptoms because influenza patients in Japan can only return to school 48 hours after fever resolution. However, we were able to confirm the usefulness of peramivir in children. Randomized case-control studies with sufficiently large populations will be conducted during the next influenza season following approval by an ethics committee.

Recent developments in antigen detection tests have made it possible to differentiate between influenza A, 2009 influenza A (H1N1), and influenza B. Therefore, on determination of the variant involved, physicians, patients, and their families can make informed decisions regarding choice of antiviral drug. The inhalation drugs laninamivir and zanamivir are difficult to use in infants. Vaccines are not completely protective because of frequent antigenic shifts in the viral surface proteins, as observed in the last pandemic.

The results of this study suggest that peramivir is an important and a feasible therapeutic option for pediatric influenza patients.

\section{Conflict of Interests}

The authors declare that they have no conflict of interests.

\section{References}

[1] A. S. Monto, I. A. Iacuzio, and J. R. LaMontagne, "Pandemic influenza: confronting a Re-emergent threat," The Journal of Infectious Diseases, vol. 176, supplement 1, pp. S1-S90, 1997.

[2] S. M. Smith and J. G. Gums, "Antivirals for influenza: strategies for use in pediatrics," Pediatric Drugs, vol. 12, no. 5, pp. 285-299, 2010.

[3] A. Örtqvist, I. Berggren, M. Insulander, B. de Jong, and B. Svenungsson, "Effectiveness of an adjuvanted monovalent vaccine against the 2009 pandemic strain of influenza $\mathrm{A}(\mathrm{H} 1 \mathrm{~N} 1) \mathrm{v}$, in Stockholm county, Sweden," Clinical Infectious Diseases, vol. 52, no. 10, pp. 1203-1211, 2011.

[4] F. G. Hayden and A. J. Hay, "Emergence and transmission of influenza A viruses resistant to amantadine and rimantadine," Current Topics in Microbiology and Immunology, vol. 176, pp. 119-130, 1992.

[5] R. G. Webster, P. A. Reay, and W. G. Laver, "Protection against lethal influenza with neuraminidase," Virology, vol. 164, no. 1, pp. 230-237, 1988.

[6] M. von Itzstein, W. Y. Wu, G. B. Kok et al., "Rational design of potent sialidase-based inhibitors of influenza virus replication," Nature, vol. 363, no. 6428, pp. 418-423, 1993.

[7] F. G. Hayden, A. D. Osterhaus, J. J. Treanor et al., "Efficacy and safety of the neuraminidase inhibitor zanamivir in the treatment of influenzavirus infections. GG167 influenza study group," The New England Journal of Medicine, vol. 337, no. 13, pp. 874-880, 1997.

[8] C. U. Kim, W. Lew, M. A. Williams et al., "Influenza neuraminidase inhibitors possessing a novel hydrophobic interaction in the enzyme active site: design, synthesis, and structural analysis of carbocyclic sialic acid analogues with potent antiinfluenza activity," Journal of the American Chemical Society, vol. 119, no. 4, pp. 681-690, 1997.

[9] N. Kawai, H. Ikematsu, N. Iwaki et al., "Factors influencing the effectiveness of oseltamivir and amantadine for the treatment of influenza: a multicenter study from Japan of the 2002-2003 influenza season," Clinical Infectious Diseases, vol. 40, no. 9, pp. 1309-1316, 2005.

[10] N. Kawai, H. Ikematsu, N. Iwaki et al., "A comparison of the effectiveness of oseltamivir for the treatment of influenza A and influenza B: a Japanese multicenter study of the 2003-2004 and 2004-2005 influenza seasons," Clinical Infectious Diseases, vol. 43, no. 4, pp. 439-444, 2006.

[11] N. Sugaya, K. Mitamura, M. Yamazaki et al., "Lower clinical effectiveness of oseltamivir against influenza B contrasted with influenza A infection in children," Clinical Infectious Diseases, vol. 44, no. 2, pp. 197-202, 2007.

[12] N. Kawai, H. Ikematsu, N. Iwaki et al., "Zanamivir treatment is equally effective for both influenza A and influenza B," Clinical Infectious Diseases, vol. 44, no. 12, p. 1666, 2007.

[13] M. Yamashita, T. Tomozawa, M. Kakuta, A. Tokumitsu, H. Nasu, and S. Kubo, "CS-8958, a prodrug of the new neuraminidase inhibitor R-125489, shows long-acting anti-influenza virus activity," Antimicrobial Agents and Chemotherapy, vol. 53, no. 1, pp. 186-192, 2009.

[14] A. Watanabe, S. C. Chang, M. J. Kim, D. W. S. Chu, and Y. Ohashi, "Long-acting neuraminidase inhibitor laninamivir octanoate versus oseltamivir for treatment of influenza: a 
double-blind, randomized, noninferiority clinical trial," Clinical Infectious Diseases, vol. 51, no. 10, pp. 1167-1175, 2010.

[15] S. Kohno, H. Kida, M. Mizuguchi, and J. Shimada, "Efficacy and safety of intravenous peramivir for treatment of seasonal influenza virus infection," Antimicrobial Agents and Chemotherapy, vol. 54, no. 11, pp. 4568-4574, 2010.

[16] N. Sugaya, "Treatment of influenza patients in their teens, side effects of oseltamivir, abnormal behavior and fall from buildings," Nipon Iji Shimpo, vol. 4360, pp. 64-68, 2007 (Japanese).

[17] J. E. Hernandez, R. Adiga, R. Armstrong et al., "Clinical experience in adults and children treated with intravenous peramivir for 2009 influenza A (H1N1) under an emergency IND program in the United States," Clinical Infectious Diseases, vol. 52, no. 6, pp. 695-706, 2011.

[18] H. Nakanishi, T. Muta, T. Fujisaki, R. Yoshitomi, T. Kubo, and E. Yokota, "Pandemic (H1N1) 2009-Associated ARDS rescued by neuraminidase inhibitors with emergency use of extracorporeal membrane oxygenation," Internal Medicine, vol. 49, no. 17, pp. 1901-1905, 2010.

[19] T. Hikita, Y. Fujii, N. Nakamoto et al., "Efficacy of metylprednisolone pulse therapy on influenza associated encephalopaty," Japanese Journal of Pediatrics, vol. 61, no. 11, pp. 2243-2249, 2008 (Japanese).

[20] N. Sugaya, D. Tamura, M. Yamazaki et al., "Comparison of the clinical effectiveness of oseltamivir and zanamivir against influenza virus infection in children," Clinical Infectious Diseases, vol. 47, no. 3, pp. 339-345, 2008. 


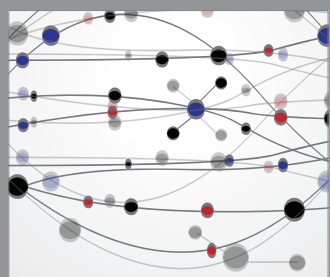

The Scientific World Journal
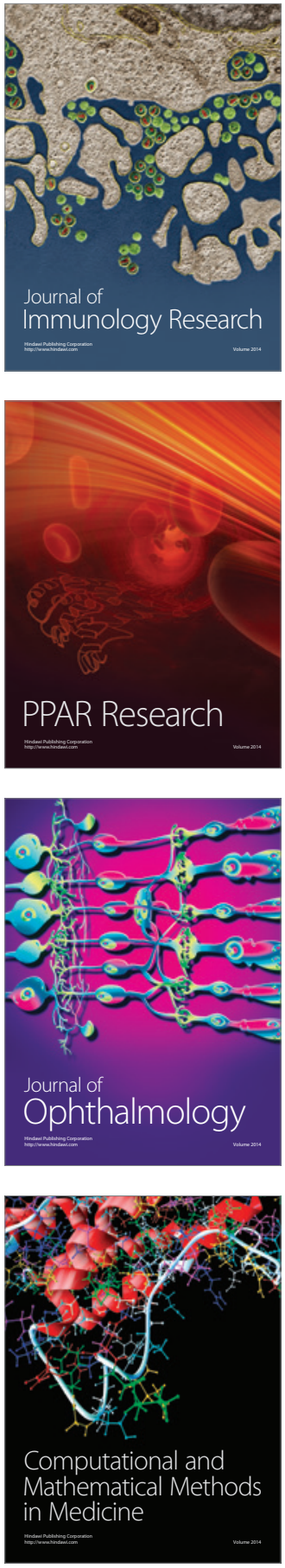

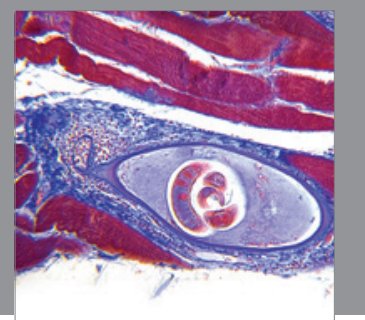

Gastroenterology

Research and Practice
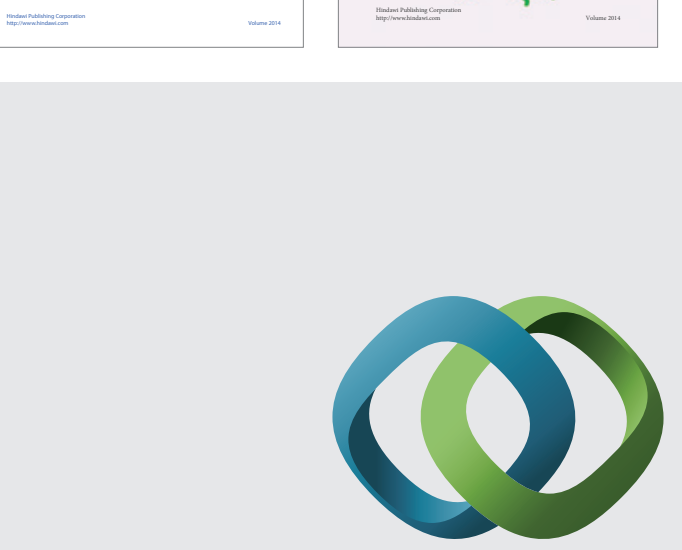

\section{Hindawi}

Submit your manuscripts at

http://www.hindawi.com
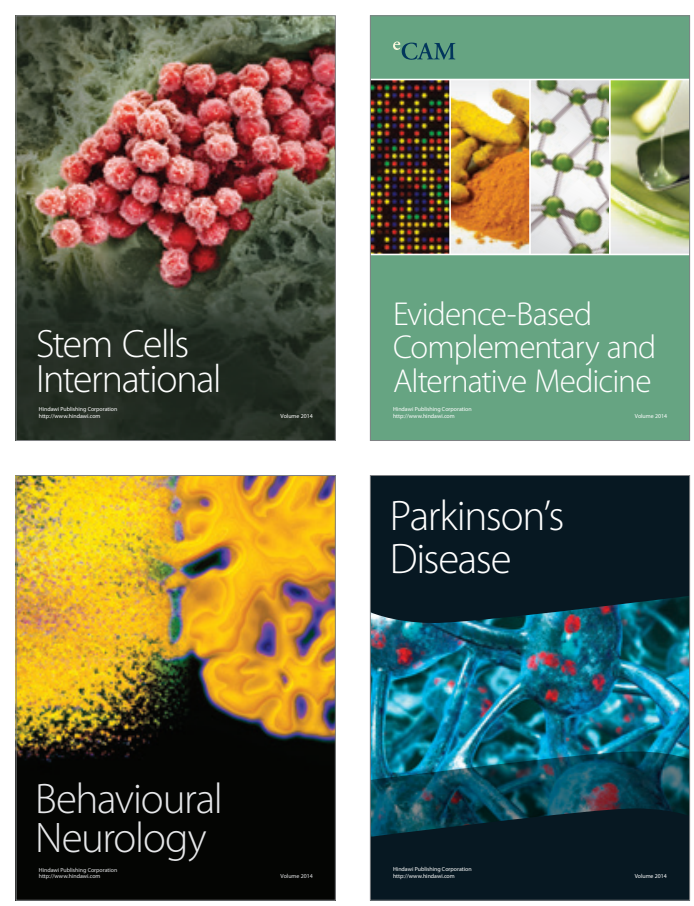

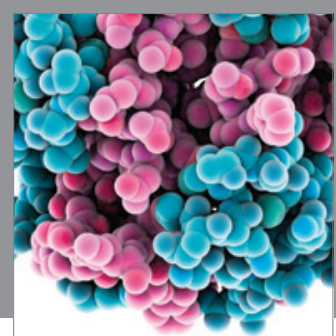

Journal of
Diabetes Research

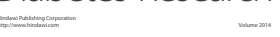

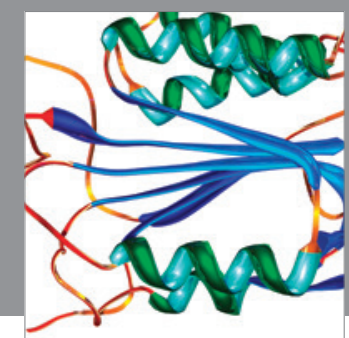

Disease Markers
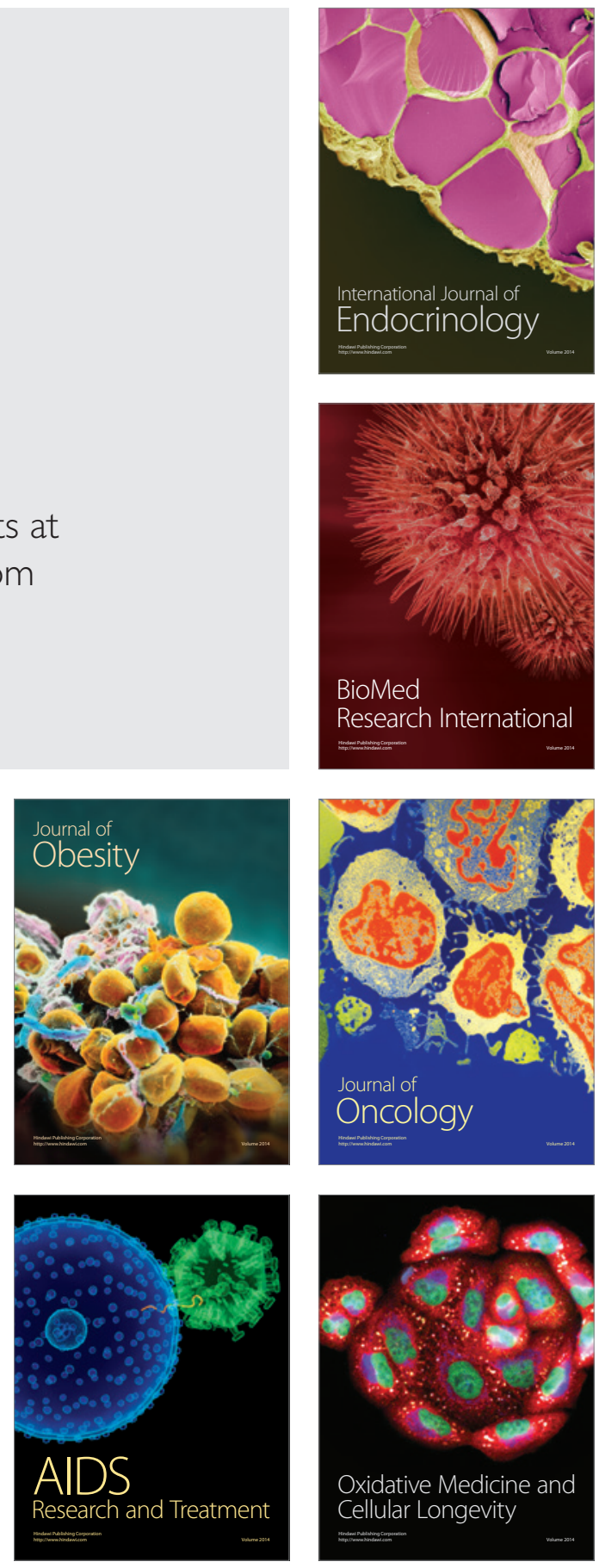